\title{
THE WIENER CLOSURE THEOREMS FOR ABSTRACT WIENER SPACES
}

\author{
J. KUELBS ${ }^{2}$ AND V. MANDREKAR ${ }^{3}$
}

\begin{abstract}
We introduce $\mathscr{L}_{1}$ and $\mathscr{L}_{2}$ translates for functions in $\mathscr{L}_{1}(\mu)$ and $\mathscr{L}_{2}(\mu)$ where $\mu$ is a Gaussian measure on a Banach space. With these translates and the Fourier-Wiener transforms defined by Cameron and Martin we obtain Wiener's closure theorem in $\mathscr{L}_{2}(\mu)$ and in $\mathscr{L}_{1}(\mu)$. Using the $\mathscr{L}_{1}(\mu)$ results we indicate the analogue of the Wiener-Pitt Tauberian theorems for this setup.
\end{abstract}

1. Introduction. Let $\mu$ be the Wiener measure on $C[0,1]$ and $\mathscr{L}_{2}(\mu)$ be the space of square integrable Borel functions with respect to $\mu$. For $f \in \mathscr{L}_{2}(\mu)$, the Fourier-Wiener transform was defined by Cameron and Martin [3]. In this paper we extend this notion to abstract Wiener spaces [6] and obtain an analogue of Wiener's closure theorem [10] for $\mathscr{L}_{2}(\mu)$. Our main effort however is to obtain an analogue of Wiener's closure theorem for $\mathscr{L}_{1}(\mu)$. From this theorem one can easily derive the Wiener-Pitt Tauberian theorem [8, p. 163].

The paper is organized as follows. In $\S 2$, we introduce the notation and sketch the extension of the Fourier-Wiener transform to abstract Wiener space. In $\S 3$ we introduce the $\mathscr{L}_{2}$-closure theorem. The results on the $\mathscr{L}_{1}$-closure theorem and the Tauberian theorem are given in the last section.

2. Preliminaries and notation. Let $H$ be a real separable Hilbert-space and suppose $\|\cdot\|_{1}$ is a measurable norm [5, p. 374]. Then it is known [6] that $\|\cdot\|_{1}$ is weaker than $\|\cdot\|$ on $H$ and the canonical Gaussian distribution on $H$ induces a Gaussian measure $\mu$ on the Borel subsets of $B$, the completion of $H$ under $\|\cdot\|_{1}$. The triple $\left(B, \mu,\|\cdot\|_{1}\right)$ is called an abstract Wiener space [6] with generating Hilbert space $H$.

Received by the editors April 12, 1971.

AMS 1970 subject classifications. Primary 28A40; Secondary 40E05.

Key words and phrases. Abstract Wiener space, Fourier-Wiener transform, Wiener closure theorems, Tauberian theorems.

${ }^{1}$ This work was done while the authors were visiting Stanford University and would like to acknowledge partial support from Professor Karlin.

${ }^{2}$ Supported in part by NSF Grant GP 9372.

${ }^{3}$ Supported in part by NSF Grant GP 28658.

(c) American Mathematical Society 1972 
If $y$ is in $B^{*}$ (the topological dual of $B$ ) then the restriction of $y$ to $H$ is continuous on $H$ because $\|\cdot\|_{1}$ is weaker than $\|\cdot\|$ on $H$. Since $H$ is dense in $B$, the restriction is a one-to-one linear map of $B^{*}$ into $H^{*}$.

We shall identify $B^{*}$ with a subset of $H^{*}$ and $H^{*}$ with $H$. Now $B^{*}$ is dense in $H^{*}=H$ since $B^{*}$ separates points of $H$, and hence $B^{*}$ is dense in $B$. Furthermore, since $B$ and $H$ are separable we have a countable set $\left\{\alpha_{n}\right\}$ of $B^{*}$ such that $\left\{\alpha_{n}\right\}$ is an orthonormal basis of $H$. Here orthogonality is with respect to inner product in $H$. For each $n,\left(x, \alpha_{n}\right), x \in B$, will mean, of course, $\alpha_{n}$ applied to the vector $x$. Since $\alpha_{n} \in B^{*} \subseteq H$ and $\left\|\alpha_{n}\right\|=1$, it follows that $\left(\cdot, \alpha_{n}\right)$ is a bounded linear functional on $B$ and that it has Gaussian distribution with mean zero and variance one with respect to the measure $\mu$ on $B$. If $h \in H$, we define

$$
(x, h)^{\sim}=\lim _{n}\left(x, h_{n}\right) \quad \text { where }\left(x, h_{n}\right)=\sum_{k=1}^{n} c_{k}\left(x, \alpha_{k}\right)
$$

and $c_{k}=\left(h, \alpha_{k}\right)$. We note that $\left\{\left(x, \alpha_{k}\right)\right\}$ is a sequence of independent Gaussian functions with mean zero and variance one, and $\sum_{k=1}^{\infty} c_{k}^{2}<\infty$, since $h \in H$ and $\left\{\alpha_{k}\right\}$ is complete orthonormal set in $H$. This implies $(x, h)^{\sim}$ exists for almost all $x \in B$ and it has a Gaussian distribution with mean zero and variance $\|h\|^{2}$. Furthermore, it is easy to show that $(x, h)^{\sim}$ equals $(x, h)$ almost everywhere on $B$ if $h \in B^{*},(x, h)^{\sim}$ is independent of the complete orthonormal set used in its definition, and finally, if $h_{1}$, $h_{2}, \cdots, h_{n}$ are orthonormal then $\left(x, h_{1}\right)^{\sim}, \cdots,\left(x, h_{n}\right)^{\sim}$ are independent Gaussian functions with mean zero and variance one.

If $f$ is a polynomial in the variables $\left\{\left(x, \alpha_{k}\right)\right\}$ then we define the FourierWiener transform $\mathscr{F}$ of $f$ following Cameron and Martin [2], [3] and Segal [9] by

$$
\mathscr{F} f(y)=\int_{B} f(\sqrt{ } 2 x+i y) d \mu(x) \quad(y \in B) .
$$

Here, if $f(x)=g\left(\left(x, \alpha_{1}\right), \cdots,\left(x, \alpha_{N}\right)\right)$ where $g$ is a function of $N$ complex variables, then $f(u+i v)=g\left[\left(u, \alpha_{1}\right)+i\left(v, \alpha_{1}\right), \cdots,\left(u, \alpha_{N}\right)+i\left(v, \alpha_{N}\right)\right]$. Then by $[2$, p. $491-492]$ or $[9$, p. 121], $\mathscr{F}$ is unitary on the class of all polynomials and

$$
\mathscr{F}^{2} f(y)=f(-y) .
$$

Hence in view of the Fourier-Hermite expansion [7, p. 436] one can extend $\mathscr{F}$ to be unitary on $\mathscr{L}_{2}(\mu)$ such that $\mathscr{F}^{2} f(y)=f(-y)$. We also remark that $\mathscr{F} f$ can be evaluated as in (2.1) for a much larger class of functionals than the polynomials indicated.

3. Wiener's theorem for $\mathscr{L}_{p}(\mu), 0<p \leqq 2$. Let $\lambda$ be the Lebesgue measure on the Borel subsets of the space of real numbers. The classical 
theorem of $\mathrm{N}$. Wiener says that any translation invariant (closed) subspace of $L_{2}(\lambda)$ consists precisely of those functions whose Fourier transforms vanish on a measurable set. Our purpose in this section is to generalize Wiener's theorem to $\mathscr{L}_{2}(\mu)$. Let $f \in \mathscr{L}_{2}(\mu)$, then for each $h \in H$, $\left(U_{h} f\right)(y)=f(y+h) \exp \left\{-\frac{1}{2}(y, h)^{\sim}-\frac{1}{4}\|h\|^{2}\right\}$ is called the $\mathscr{L}_{2}$-translate of $f$. It is easy to check by the translation theorem as given in [7, p. 435] that, for each $h \in H, U_{h}$ is a unitary operator on $\mathscr{L}_{2}(\mu)$ onto $\mathscr{L}_{2}(\mu)$. The main tool of the classical proof is the relation of the Fourier transform of the translate of a function to the Fourier transform of the function itself. The following lemma gives the analogous relation for $f \in \mathscr{L}_{2}(\mu)$.

LEMMA 3.1. Let $f \in \mathscr{L}_{2}(\mu)$, then, for each $h \in H$,

with $\mu$-measure one on $B$.

$$
\mathscr{F}\left(U_{h} f\right)(y)=\exp \left\{-\frac{i}{2}(y, h)^{\sim}\right\} \mathscr{F} f(y)
$$

Proof. We first assume that $f$ is a polynomial in some of the variables $\left\{\left(x, \alpha_{k}\right): k \geqq 1\right\}$ where $\left\{\alpha_{k}\right\}$ is completely orthonormal in $B^{*} \subseteq H^{*}$. Then for $h \in H$ we obtain

$$
\begin{aligned}
\mathscr{F} f(y)= & \int_{B} f(\sqrt{ } 2 x+i y) d \mu(x) \\
= & \int_{B} f\left(\sqrt{ } 2\left(x+\frac{h}{\sqrt{ } 2}\right)+i y\right) \exp \left\{-\left(x, \frac{h}{\sqrt{ } 2}\right)^{\sim}-\frac{(h, h)}{4}\right\} d \mu(x) \\
= & \int_{B} f(\sqrt{ } 2 x+i y+h) \exp \left\{-\frac{1}{2}(\sqrt{ } 2 x+i y, h)^{\sim}-\frac{(h, h)}{4}\right\} d \mu(x) \\
& \cdot \exp \left\{\frac{i}{2}(y, h)\right\} \\
= & \int_{B}\left(U_{h} f\right)(\sqrt{ } 2 x+i y) d \mu(x) \cdot \exp \left\{\frac{i}{2}(y, h)^{\sim}\right\} \\
= & \exp \left\{\frac{i}{2}(y, h)^{\sim}\right\} \mathscr{F}\left(U_{h} f\right)(y) .
\end{aligned}
$$

Here the translation by $h \in H$ is handled as indicated due to the translation theorem [7, p. 435].

For arbitrary $f$ in $\mathscr{L}_{2}(\mu)$ we proceed as follows. The operators $U_{h}$ and $\mathscr{F}$ are unitary operators on $\mathscr{L}_{2}(\mu)$ such that, for each $h \in H$ and polynomial $f$ in $\left\{\left(x, \alpha_{k}\right)\right\}$, we have the equation

$$
\mathscr{F}\left(U_{h} f\right)=e_{h}(\cdot) \mathscr{F} f
$$

where $e_{h}(\cdot)=\exp \left\{-i(\cdot, h)^{\sim} / 2\right\}$ and the equation is understood as $\mathscr{L}_{2^{-}}$ equivalence. Since such polynomials are dense in $\mathscr{L}_{2}(\mu)[7, \mathrm{p} .436]$, the proof follows. 
The following is our version of Wiener's theorem.

THEOREM 3.2. Let $\mathfrak{W}$ be a translation invariant closed subspace of $\mathscr{L}_{2}(\mu)$; i.e., $U_{h} \mathfrak{W} \subseteq \mathfrak{W}$, for each $h \in H$. Then there exists a measurable subset $E$ of $B$ such that $\mathfrak{B}=M_{E}$ where $M_{E}=\left\{f \mid f \in \mathscr{L}_{2}(\mu)\right.$ such that $\mathscr{F} f(y)=0$ a.e. $[\mu]$ for all $y \in E\}$. Conversely, each $M_{E}$ is translation invariant. Further, $M_{A}=M_{B}$ if and only if $\mu(A \triangle B)=0$.

Proof. The converse being obvious from Lemma 3.1 we proceed to the direct part. We note that the proof is basically classical. Let $\mathfrak{B}$ be a translation invariant closed subspace of $\mathscr{L}_{2}(\mu)$. Let $\mathfrak{N}=\mathscr{F} \mathfrak{W}$. Since $\mathscr{F}$ is unitary, $\mathfrak{N}$ is a closed subspace of $\mathscr{L}_{2}(\mu)$ and in view of Lemma 3.1, $\mathfrak{N}$ is invariant under multiplication by $e_{h}(\cdot)$. Let $P$ be the orthogonal projection of $\mathscr{L}_{2}(\mu)$ onto $\mathfrak{N}$. Then $f-P f \perp P g$ for all $f, g \in \mathscr{L}_{2}(\mu)$ and since $\mathfrak{N}$ is invariant under multiplication by $e_{h}(\cdot)$, we have, for all $h \in H$,

$$
\int_{B}(f(x)-(P f)(x)) \overline{(P g)(x)} e_{-h}(x) d \mu(x)=0 .
$$

Since $B^{*} \subset H, B$ is separable, and every (complex) measure on $B$ is uniquely determined by its Fourier transform, the above equation implies that, for all $f, g \in \mathscr{L}_{2}(\mu)$,

$$
f(x)(P g)(x)=(P f)(x)(P g)(x) \quad \text { a.e. } \mu \text {. }
$$

Interchanging the roles of $f$ and $g$ we obtain

$$
f(x)(P g)(x)=g(x)(P f)(x) \text { a.e. } \mu .
$$

Taking $g \equiv 1$ we get

$$
(P f)(x)=\varphi(x) f(x) \quad \text { a.e. } \mu \text { for all } f \in \mathscr{L}_{2}(\mu),
$$

where $\varphi(x)$ is the projection of the function identically one onto $\mathfrak{N}$. But $P^{2}=P$ implies $\varphi^{2}=\varphi$ a.e. $\mu$. Hence $\varphi(x)=0$ or 1 a.e. $\mu$ and we let $E=\{x ; \varphi(x)=0\}$. Since $f \in \mathfrak{N}$ iff $f=P f=\varphi \cdot f$ we see that $\mathfrak{N}$ consists of those functions which vanish at least on $E$, giving $\mathfrak{B}=M_{E}$. The uniqueness part being simple is omitted.

Corollary 3.1 (WIENer [10, p. 267]). Let $f \in \mathscr{L}_{2}(\mu)$ such that $\mu\{y ;(\mathscr{F} f)(y)=0\}=0$. Then the linear manifold generated by the $\mathscr{L}_{2^{-}}$ translates of $f$ is dense in $\mathscr{L}_{p}(\mu), 0<p \leqq 2$.

Proof. Since $\mathscr{L}_{2}(\mu)$ is dense in $\mathscr{L}_{p}(\mu)$ is suffices to show the theorem in the case $p=2$. Let $\mathfrak{B}$ be the closed subspace of $\mathscr{L}_{2}(\mu)$ generated by the $\mathscr{L}_{2}$-translates of $f$. Then clearly $\mathfrak{B}$ is translation invariant and since $\mathscr{F} f(y) \neq 0$ with $\mu$-measure one $\mathfrak{W}=M_{\varnothing}$ where $\varnothing$ is the empty set. By definition $M_{\varnothing}=\mathscr{L}_{2}(\mu)$ so the proof is complete. 
4. Translation in $\mathscr{L}_{1}(\mu)$ and a Tauberian theorem. The translation operation $U_{h} f(h \in H)$ as used previously is an isometry on $\mathscr{L}_{2}(\mu)$ onto $\mathscr{L}_{2}(\mu)$, but it is not an isometry in $\mathscr{L}_{1}(\mu)$ unless one has the trivial situation $h=0$. The translation for functions in $\mathscr{L}_{1}(\mu)$ which is an isometry is the following:

$$
W_{h} f(x)=f(x+h) \exp \left\{-(x, h)^{\sim}-\frac{1}{2}(h, h)\right\} \quad(h \in H) .
$$

That it is an isometry from $\mathscr{L}_{1}(\mu)$ onto $\mathscr{L}_{1}(\mu)$ follows easily from the translation theorem of Cameron-Martin for this setting [7, p. 435]. As we shall see later it also behaves nicely with respect to convolution.

Now the Fourier-Wiener transform of the translate $W_{h} f$ (assuming $f$ is in $\mathscr{L}_{2}(\mu)$ so that $\mathscr{F} f$ is defined) does not retain the crucial property of Lemma 3.1. However, if we use a slightly modified Fourier-Wiener transform we can obtain a similar result.

We now define the $\mathscr{L}_{1}$-Fourier-Wiener transform of a function $f \in \mathscr{L}_{1}(\mu)$ by

$$
\mathscr{F}_{1} f(h)=\int_{B} \exp \left\{i(x, h)^{\sim}\right\} f(x) \mu(d x) \quad \text { for } h \in H .
$$

In view of the translation theorem [7, p. 435] we have, for each $h_{0} \in H$,

$$
\mathscr{F}_{1}\left(W_{h_{0}} f\right)(h)=\exp \left\{-i\left(h_{0}, h\right)\right\} \mathscr{F}_{1} f(h) \quad \text { for } h \in H .
$$

We are now ready to prove a theorem for the $\mathscr{L}_{1}$-transform and $\mathscr{L}_{1^{-}}$ translates. As one might guess we make use of Wiener's original theorem in some way. We also point out that the $\mathscr{L}_{1}$-transform in (4.2) is closely related to the Fourier-Wiener transform of Cameron and Martin in [2] which was subsequently modified in [3] becoming the $\mathscr{L}_{2}$-transform.

A function $f$ is a tame function on $B$ if there exist vectors $h_{1} h_{2}, \cdots$, $h_{k} \in H$ such that

$$
f(x)=\Gamma\left(\left(x, h_{1}\right)^{\sim}, \cdots,\left(x, h_{k}\right)^{\sim}\right)
$$

where $\Gamma\left(u_{1}, u_{2}, \cdots, u_{k}\right)$ is a Borel measurable function on $R_{k}$. By orthogonalization of $h_{1}, h_{2}, \cdots, h_{k}$ we can always write a tame function in the form

$$
f(x)=\Psi\left(\left(x, \varphi_{1}\right)^{\sim}, \cdots,\left(x, \varphi_{N}\right)^{\sim}\right)
$$

where $\varphi_{1}, \cdots, \varphi_{N}$ are orthonormal in $H$ and $\Psi$ is Borel measurable on $R_{N}$. Hence we lose no generality in assuming (4.4) is the case.

THEOREM 4.1. Let $f \in \mathscr{L}_{1}(\mu)$ be of the form (4.4) and $\mathscr{F}_{1} f(h) \neq 0$ for all $h \in H$. Then $\mathfrak{W}_{f}$, the linear manifold generated by the $\mathscr{L}_{1}$-translates of $f$, is dense in $\mathscr{L}_{p}(\mu)$ for $0 \leqq p \leqq 1$. 
Proof. Let $\left\{\varphi_{j}: j \geqq N+1\right\}$ be an orthonormal set in $H$ such that $\left\{\varphi_{j}: j \geqq 1\right\}$ is complete. Then, using the Fourier-Hermite expansion of functions in $\mathscr{L}_{2}(\mu)$ due to Cameron and Martin [4] and appearing in this generality in Lemma 2.2 of [7], we see that tame functions of the form

$$
L(x)=\Phi\left[\left(x, \varphi_{1}\right)^{\sim}, \cdots,\left(x, \varphi_{m}\right)^{\sim}\right] \quad(m=1,2, \cdots)
$$

where $L \in \mathscr{L}_{2}(\mu)$ are dense in $\mathscr{L}_{2}(\mu)$. Since each element in $\mathscr{L}_{1}(\mu)$ can be approximated in $\mathscr{L}_{1}$-norm by a function in $\mathscr{L}_{2}(\mu)$ and the $\mathscr{L}_{2}(\mu)$-norm is greater than the $\mathscr{L}_{1}$-norm on $\mathscr{L}_{2}(\mu)$ it follows that functions of the form (4.5) are dense in $\mathscr{L}_{1}(\mu)$ with respect to the $\mathscr{L}_{1}$-norm. Hence the theorem is proved if the $\mathscr{L}_{1}$-translates approximate any function $L \in \mathscr{L}_{2}(\mu)$ which is of the form (4.5).

Now any tame function of the form (4.5) with $m<N$ can be written as a tame function with $m=N$ by simply multiplying $\Phi\left(u_{1}, \cdots, u_{m}\right)$ by $\Phi_{1}\left(u_{n+1}, \cdots, u_{N}\right) \equiv 1$ and hence we can assume $m \geqq N$. On the other hand, if $m>N$ we then write

$$
f(x)=\Psi\left[\left(x, \varphi_{1}\right)^{\sim}, \cdots,\left(x, \varphi_{N}\right)^{\sim}\right] \cdot \Phi_{2}\left[\left(x, \varphi_{N+1}\right)^{\sim}, \cdots,\left(x, \varphi_{m}\right)^{\sim}\right]
$$

where $\Phi_{2}\left(u_{N+1}, \cdots, u_{m}\right) \equiv 1$. Hence we can assume without loss of generality that $m=N$.

By assumption we have for $h$ of the form $\sum_{i=1}^{N} a_{i} \varphi_{i},\left(a_{1}, a_{2}, \cdots, a_{N}\right) \in$ $R_{N}$,

$$
\mathscr{F}_{1} f(h)=\int_{H} \exp \left\{i(x, h)^{\sim}\right\} f(x) \mu(d x) \neq 0 .
$$

Hence we have

$$
\begin{aligned}
\mathscr{F}_{1} f(h)=(2 \pi)^{-N / 2} \int_{R^{N}} \exp \left\{i \sum_{i=1}^{N} a_{i} u_{i}\right\} & \Psi\left[\left(u_{1}, \cdots, u_{N}\right)\right] \\
& \times \exp \left\{-\frac{1}{2} \sum_{i=1}^{N} u_{i}^{2}\right\} d u_{1}, \cdots, d u_{N} \neq 0 .
\end{aligned}
$$

Thus the ordinary Fourier transform of

$$
\Lambda(v)=(2 \pi)^{-N / 2} \Psi(v) \exp \left\{-\frac{1}{2} v \cdot v\right\} \quad\left(v \in R_{N}\right)
$$

never vanishes on $R_{N}$. Here we use $v \cdot u$ to denote $\sum_{1}^{N} u_{j} v_{j}$ if $u, v \in R_{N}$. Thus the ordinary translates of (4.7) generate a dense subset of $L_{1, N}$ where $L_{1, N}$ denotes the integrable Borel functions with respect to Lebesgue measure on $R_{N}$. Let $L_{1, N}^{g}$ denote the Borel functions on $R_{N}$ which are integrable with respect to the Gaussian density

$$
g(v)=(2 \pi)^{-N / 2} \exp \left\{-\frac{1}{2} v \cdot v\right\} \quad\left(v \in R_{N}\right) .
$$

Take an arbitrary tame function $L \in \mathscr{L}_{2}(\mu)$ of the form (4.5) with $m=N$. 
Then $\Phi(v) \in L_{1, N}^{g}$ and $\Phi(v) g(v) \in L_{1, N}$. Take $\varepsilon>0$. Then by Wiener's theorem for $L_{1, N}\left[8\right.$, p. 162] there exist translates $t_{1}, \cdots, t_{k} \in R_{N}$ and constants $c_{1}, \cdots, c_{k}$ such that

$$
\int_{R_{N}}\left|\sum_{j=1}^{K} c_{j} \Lambda\left(v+t_{j}\right)-\Phi(v) g(v)\right| d v<\varepsilon .
$$

Using (4.7), (4.8), and (4.9) we see that

$$
\int_{R_{N}}\left|\sum_{j=1}^{K} c_{j} \Psi\left(v+t_{j}\right) \exp \left\{-v \cdot t_{j}-\frac{1}{2} t_{j} \cdot t_{j}\right\}-\Phi(v)\right| g(v) d v<\varepsilon
$$

Choosing $h_{1}, \cdots, h_{k}$ in the subspace of $H$ generated by $\left\{\varphi_{1}, \cdots, \varphi_{N}\right\}$ and such that $t_{j}=\left[\left(h_{j}, \varphi_{1}\right), \cdots,\left(h_{j}, \varphi_{N}\right)\right](j=1, \cdots, K),(4.10)$ then implies

$$
\int_{B}\left|\sum_{j=1}^{K} c_{j} W_{h_{j}} f(x)-L(x)\right| d \mu(x)<\varepsilon .
$$

Hence the tame functions in $\mathscr{L}_{2}(\mu)$ of the form (4.5) with $m=N$ can be approximated in $\mathscr{L}_{1}$-norm by our $\mathscr{L}_{1}$-translates of $f$. Since $m=N$ represents the general case $\mathfrak{W}_{f}$ is dense in $\mathscr{L}_{1}(\mu)$. The proof is now complete since $\mathscr{L}_{1}(\mu)$ is dense in $\mathscr{L}_{p}(\mu)$ and the $\mathscr{L}_{1}$-norm dominates the $\mathscr{L}_{p}$-distance, $0<p \leqq 1$.

The function $f \in \mathscr{L}_{1}(\mu)$ is said to be splittable with respect to the complete orthonormal set $\left\{\varphi_{k}\right\}$ if there exists a sequence of integers $N_{1}<N_{2}<\cdots$ such that, for each integer $k$,

$$
f(x)=L_{k}(x) \cdot \Gamma_{k}(x) \text { a.e. }[\mu]
$$

where $L_{k}(x)=\Phi_{k}\left[\left(x, \varphi_{1}\right)^{\sim}, \cdots,\left(x, \varphi_{N_{k}}\right)^{\sim}\right]$ and $\Gamma_{k}$ is $\mathscr{B}_{k}$ measurable on $B$ where $\mathscr{B}_{k}$ is the minimal $\sigma$-algebra generated by the functionals $\left\{\left(\cdot, \varphi_{j}\right)^{\sim}\right.$, $\left.j \geqq N_{k}+1\right\}$.

Remark 4.1. Since $\Gamma_{k}$ is $\mathscr{B}_{k}$-measurable on $B$ it follows [1, p. 395] that there exists a Borel measurable function defined on the space of all real sequences such that

$$
\Gamma_{k}(x)=F\left(\left(x, \varphi_{N_{K}+1}\right)^{\sim}, \cdots\right) \text { a.e. }[\mu] .
$$

Also from (4.12) and the fact that $f \in \mathscr{L}_{1}(\mu)$ we get that $L_{k}, \Gamma_{k}$ are in $\mathscr{L}_{1}(\mu)$ provided $f \not \equiv 0$.

We say that $f \in \mathscr{L}_{1}(\mu)$ is negligibly split if $f$ is splittable and for every $\varepsilon>0$, there exists a $k$ such that

$$
\int_{B}\left|\Gamma_{k}(x)-1\right| d \mu(x)<\varepsilon .
$$

It is easy to see that $f$ is then the product of tame functions. 
THEOREM 4.2. Let $f \in \mathscr{L}_{1}(\mu)$ be negligibly split with respect to the complete orthonormal bases $\left\{\varphi_{k}\right\}$ in $H$ and assume that $\left(\mathscr{F}_{1} f\right)(h) \neq 0$ for all $h \in H$. Then the linear manifold $\mathfrak{W}_{\text {f }}$ generated by $\mathscr{L}_{1}$-translates of $f$ is dense in $\mathscr{L}_{p}(\mu), 0<p \leqq 1$.

Proof. In view of the argument given in the proof of Theorem 4.1 it suffices to prove that one can approximate in $\mathscr{L}_{1}$-norms functions $L \in \mathscr{L}_{2}(\mu)$ of the form (4.5). Take $\varepsilon>0$, and suppose $L(x)$ is given. Then there exists $N_{k}$ such that $N_{k} \geqq m$ and

$$
\int_{B}\left|\Gamma_{N_{k}}(x)-1\right| d \mu(x)<\varepsilon .
$$

Again arguing as in Theorem 4.1 we can now assume that $m=N_{k}$. Also we have

$$
\mathscr{F}_{1} f(y)=\mathscr{F}_{1} L_{k}(y) \mathscr{F}_{1} \Gamma_{k}(y) e^{(y, v) / 2}, \quad y \in H .
$$

The above equation holds as indicated since the functionals $L_{k}$ and $\Gamma_{k}$ are independent (probabilistic sense). Since $\mathscr{F}_{1} f(y) \neq 0$ we get $\mathscr{F}_{1} L_{k}(y) \neq 0$ by (4.14). Now by the proof of Theorem 4.1 there exist constants $c_{1}, c_{2}, \cdots, c_{r}$ and vectors $h_{1}, h_{2}, \cdots, h_{r}$ in the subspace of $H$ generated by $\left\{\varphi_{1}, \cdots, \varphi_{N_{k}}\right\}$ such that

$$
\int_{B}\left|\sum_{j=1}^{r} c_{j} W_{h_{j}} L_{k}(x)-L(x)\right| d \mu(x)<\varepsilon .
$$

In view of Remark 4.1 it follows that

and hence

$$
\Gamma_{k}\left(x+h_{j}\right)=\Gamma_{k}(x) \quad(j=1, \cdots, r)
$$

$$
W_{h_{j}} f(x)=\left[W_{h_{j}} L(x)\right] \cdot \Gamma_{k}(x) \quad(j=1, \cdots, r) .
$$

Now $\Gamma_{k}$ and $L_{k}$ are independent (probabilistic sense) thus (4.13) and (4.15) imply

$$
\begin{aligned}
\int_{B} \mid \sum_{j=1}^{r} c_{j} W_{h_{j}} f(x) & -\sum_{j=1}^{r} c_{j} W_{h_{j}} L_{k}(x) \mid d \mu(x) \\
& =\int_{B}\left|\sum_{j=1}^{r} c_{j} W_{h} L_{k}(x)\left[\Gamma_{k}(x)-1\right]\right| d \mu(x) \\
& =\int_{B}\left|\sum_{j=1}^{r} c_{j} W_{h j} L_{k}(x)\right| d \mu(x) \cdot \int_{B}\left|\Gamma_{k}(x)-1\right| d \mu(x) \\
& \leqq\left[\int_{B}|L(x)| d \mu(x)+\varepsilon\right] \times \varepsilon .
\end{aligned}
$$


Combining (4.15) and (4.16) along with $\varepsilon>0$ being arbitrary completes the proof.

REMARK 4.2. If $f$ is a tame function of the form (4.4) then $f$ is easily seen to be negligibly split with respect to the complete orthonormal basis $\left\{\varphi_{k}\right\}$ where $\left\{\varphi_{1}, \cdots, \varphi_{N}\right\}$ are as in (4.4). Thus Theorem 4.2 actually implies Theorem 4.1, but we proved both theorems since a direct proof of Theorem 4.2 would involve about the same amount of effort.

For an example of a function $f \in \mathscr{L}_{1}(\mu)$ which satisfies Theorem 4.2 but not Theorem 4.1 consider

$$
f(x)=\exp \left\{\sum_{k=1}^{\infty} \lambda_{k}\left(x, \varphi_{k}\right)^{\sim 2}\right\}
$$

where $\left\{\lambda_{k}\right\}$ is a sequence of positive numbers such that $\sum_{k=1}^{\infty} \lambda_{k}<\frac{1}{2}$ and $\left\{\varphi_{k}\right\}$ is an orthonormal set in $H$. Then $f \in \mathscr{L}_{1}(\mu)$,

$$
\mathscr{F}_{1} f(h)=\exp \left\{-\sum_{k=1}^{\infty}\left(h, \varphi_{k}\right)^{2} / 2\left(1-2 \lambda_{k}\right) \neq 0\right.
$$

and $f$ is negligibly split with respect to $\left\{\varphi_{k}\right\}$.

If $f$ and $\varphi$ are measurable functions on $B$ we define the convolution of $f$ and $\varphi$ by the usual formula

$$
f * \varphi(x)=\int_{B} f(y) \varphi(y-x) d \mu(y) .
$$

In this setup convolution is not always commutative since the measure $\mu$ is not translation invariant. It does, however, act in a normal way with respect to translation if we use $\mathscr{L}_{1}$-translates and we show this in the next lemma.

Lemma 4.3. Suppose $f \in \mathscr{L}_{1}(\mu)$ and $\varphi \in \mathscr{L}_{\alpha}(\mu)$. Then

(1) $f * \varphi(x)$ exists for each $x$ in $B$,

(2) $\left(W_{h} f\right) * \varphi(x)=f * \varphi(x+h)$ for each $x \in B$ and $h \in H$.

Proof. Since $\varphi$ is in $\mathscr{L}_{\infty}(\mu)$ and $\varphi(y-x)$ is measurable as a function of $y$ for each $x \in B$ the conclusion of (1) is immediate. Now $f \in \mathscr{L}_{1}(\mu)$ iff $W_{h} f \in \mathscr{L}_{1}(\mu)$ for each $h \in H[7$, p. 435$]$ so it follows that $W_{h} f * \varphi(x)$ exists on $B$ for each $x \in H$. Further,

$$
\begin{aligned}
W_{h} f * \varphi(x) & =\int_{B} W_{h} f(y) \varphi(y-x) d \mu(y) \\
& =\int_{B} f(y+h) \exp \left\{-(x, h)^{\sim}-\frac{1}{2}(h, h)^{\sim}\right\} \varphi(y-x) d \mu(y) \\
& =\int_{B} f(y) \varphi(y-x-h) d \mu(y)=f * \varphi(x+h)
\end{aligned}
$$


where the third equality follows from the translation theorem [7, p. 435]. Hence (2) holds.

We now state a Tauberian theorem for abstract Wiener spaces. Its proof is exactly as in $[10$, p. 285] if one uses the definition of convolution in (4.17) and the $\mathscr{L}_{1}$-translates. If $\varphi$ is defined on $B$ we say $\lim _{x \rightarrow \infty} \varphi(x)=c$ if for each $\varepsilon>0$ there exists a bounded set $E$ such that $|\varphi(x)-c|<\varepsilon$ on $B-E$.

TheOREM 4.3. Suppose $f \in \mathscr{L}_{1}(\mu)$ and $\mathscr{F}_{1} f(y) \neq 0, y \in H$. Further, assume $\varphi \in \mathscr{L}_{\infty}(\mu)$ and $c$ is a constant such that

$$
\lim _{x \rightarrow \infty} f * \varphi(x)=c \mathscr{F}_{1} f(0) .
$$

Then, if $f$ is negligibly split, we have $\lim g * \varphi(x)=c \int_{B} g(x) d \mu(x)$ for all $g \in \mathscr{L}_{1}(\mu)$.

As a final remark we mention that Pitt's Tauberian theorem, as it appears in [8, p. 163], can also be proved in this setting. Here, however, we would define slowly oscillating in terms of a norm bounded set and a norm bounded neighborhood of zero. Since open subsets of $B$ have positive $\mu$-measure the proof is as in [8].

\section{REFERENCES}

1. Leo Brieman, Probability, Addison-Wesley, Reading, Mass., 1968. MR 37 \#4841.

2. R. H.Cameron and W. T. Martin, Fourier-Wiener transforms of analytic functionals, Duke Math. J. 12 (1945), 489-507. MR 7, 62.

3. - Fourier-Wiener transforms of functionals belonging to $L_{2}$ over the space $C$, Duke Math. J. 14 (1947), 99-107. MR 8, 523.

4. - The orthogonal development of non-linear functionals in series of FourierHermite functionals, Ann. of Math. (2) 48 (1947), 385-392. MR 8, 523.

5. L. Gross, Measurable functions on Hilbert space, Trans. Amer. Math. Soc. 105 (1962), 372-390. MR 26 \#5121.

6. - Abstract Wiener spaces, Proc. Fifth Berkeley Sympos. Math. Statist. and Probability (Berkeley, Calif., 1965/66), vol. II: Contributions to Probability Theory, part 1, Univ. of California Press, Berkeley, Calif., 1967, pp. 31-42. MR 35 \#3027.

7. J. Kuelbs, Abstract Wiener spaces and applications to analysis, Pacific J. Math. 31 (1969), 433-450. MR 40 \#5817.

8. W. Rudin, Fourier analysis on groups, Interscience Tracts in Pure and Appl. Math., no. 12, Interscience, New York, 1962. MR 27 \#2808.

9. I. E. Segal, Tensor algebras over Hilbert spaces. I, Trans. Amer. Math. Soc. 81 (1956), 106-134. MR 17, 880.

10. N. Wiener, Selected papers, M.I.T. Press, Cambridge, Mass., 1964, pp. 261-360. MR 32 \#3.

Department of Mathematics, University of Wisconsin, Madison, Wisconsin 53706

Department of Statistics and Probability, Michigan State University, East Lansing, Michigan 48823 